\title{
Menopausal Hormone Therapy for Cardiovascular Protection-Unfulfilled Expectations
}

\author{
Nanette K. Wenger
}

Received: 29 April 2009/Accepted: 26 May 2009/Published online: 10 June 2009

(C) Springer Science + Business Media, LLC 2009

\begin{abstract}
Despite biologically plausible mechanisms of benefit and data from observational studies suggesting cardioprotection from menopausal hormone therapy, the results of randomized clinical trials in both healthy women and women with established coronary heart disease failed to show benefit and indeed imparted some harm. In 2009, menopausal hormone therapy is not indicated for the primary prevention of coronary heart disease or of cardiovascular disease. Prevention of cardiovascular disease in women should be addressed by the aggressive identification and modification of established cardiovascular risk factors. Much remains unknown about the mechanisms of menopausal hormone therapy action and the issue of whether there is a therapeutic window of opportunity for its use.
\end{abstract}

Keywords Hormone Therapy · Estrogen ·

Cardiovascular Protection · Clinical Trials

Epidemiologic data [1] have demonstrated an association between postmenopausal estrogen use and reduction in the risk of coronary heart disease, postulated as mediated by a favorable effect of estrogen on traditional coronary risk

\footnotetext{
N. K. Wenger

Grady Memorial Hospital, Atlanta, GA, USA

N. K. Wenger

Emory Heart and Vascular Center,

Atlanta, GA, USA

N. K. Wenger $(\bowtie)$

Emory University School of Medicine,

49 Jesse Hill Jr. Drive,

Atlanta, GA, USA

e-mail: nwenger@emory.edu
}

factors [2]. Biologically plausible mechanisms for cardioprotection include favorable lipid effects; favorable effects on coagulation, fibrinolysis, and homocysteine; decreased inflammatory response to atherosclerosis; decreased vascular smooth muscle proliferation; promotion of endotheliumdependent vascular dilation and decreased thrombosis; lessened LDL oxidation; and promotion of angiogenesis, among others. But biologic plausibility alone is insufficient evidence for cardioprotection. Why, then, have randomized clinical trials of menopausal hormone therapy failed to show benefit and indeed imparted harm? Hormone users in the observational studies tended to be healthier, wealthier, and have fewer coronary risk characteristics. A 2002 systematic review and meta-analysis of menopausal hormone therapy for the primary prevention of cardiovascular disease displayed that adjustment of these observational studies for socioeconomic status, educational level, and major coronary risk factors failed to support the use of such therapy for the primary prevention of cardiovascular and coronary heart disease [3].

What data derive from the major randomized clinical trials? The Heart and Estrogen/Progestin Replacement Study (HERS) allocated 2,763 menopausal women with a uterus and with documented coronary heart disease to $0.625 \mathrm{mg}$ oral conjugated estrogen (CEE) plus $2.5 \mathrm{mg}$ medroxyprogesterone acetate (MPA) daily vs. placebo. The primary outcome was the combined endpoint of nonfatal myocardial infarction or coronary death. Surveillance at 6.8 years in HERS and HERS II [4, 5] showed no reduction in the primary endpoint or in the risk of cardiovascular events but a pattern of early increased risk of coronary events. Adverse events included an increased risk of venous thromboembolism and of biliary tract surgery without significant effect on breast cancer, any cancer, hip fracture, or any fracture. The HERS investigators concluded that 
menopausal hormone therapy should not be used to reduce coronary risk in women with coronary heart disease.

Data for healthy women (no clinical evidence of cardiovascular disease) derived from the Women's Health Initiative (WHI) randomized estrogen/progestin (E/P) study [6] and the unopposed E study [7] which recruited 16, 608 women in the $\mathrm{E} / \mathrm{P}$ study and 10,739 women after hysterectomy in the E study, all aged 50-79 years (mean age 63 years). E/P administered $0.625 \mathrm{mg}$ oral CEE plus $2.5 \mathrm{mg}$ MPA daily vs. placebo and E $0.625 \mathrm{mg}$ oral CEE daily vs. placebo.

The E/P trial was terminated prematurely after an average follow-up of 5.2 years for health safety concerns, namely an increased risk of invasive breast cancer and an unfavorable global risk score. Despite a low absolute risk of harm for an individual woman, there was a $26 \%$ increased risk of invasive breast cancer, a $29 \%$ increase in coronary events, a $41 \%$ increased stroke risk, and a doubled risk of venous thromboembolism. There was an $81 \%$ excess risk of myocardial infarction in the first year of hormone therapy. Benefits included a $37 \%$ decreased risk of colorectal cancer, a $33 \%$ decrease in hip fracture risk, and a $24 \%$ decrease in risk for total fracture; all-cause mortality was unchanged. The population risk was considered substantial and not consistent with requirements for primary prevention. Question has been raised as to the baseline risk profile of WHI women and whether some or all characteristics of the metabolic syndrome contributed to the adverse outcome of menopausal hormone therapy. Additionally, a memory substudy in 4,532 WHI women aged 65 years and older showed a doubled risk of probable dementia with hormone therapy and lack of prevention of mild cognitive decline [8].

The WHI E arm was also discontinued prematurely after nearly 7 years of follow-up due to an increase in stroke risk similar to that with E/P and lack of improvement in the global risk score. There was an unchanged heart-disease risk, a decreased risk for hip fracture, a nonsignificant decrease in breast cancer risk, no decrease in the risk of colon cancer, and a trend to increased risk for probable dementia and/or mild cognitive impairment. The WHI investigators concluded that these regimens of menopausal hormone therapy should not be initiated or continued for the primary prevention of coronary heart disease.

Three years after stopping treatment in the WHI E/P trial [9], the increased risk for MI, stroke, and venous thromboembolism disappeared. The increased risk for breast and other cancers persisted, mandating continued surveillance, and improvement in bone health disappeared, mandating surveillance for osteoporosis. The global risk index remained $12 \%$ increased for $\mathrm{E} / \mathrm{P}$ vs. placebo.

Study of the selective estrogen receptor modulator raloxifene [10] in the Raloxifene Use for The Heart
(RUTH) trial failed to show benefit in 10,101 women with coronary risk factors or with established coronary heart disease despite decreasing the risk for invasive breast cancer. Venous thromboembolism was increased, and there was an increase in stroke death without an increase in stroke.

Recently, the US FDA warned pharmacy operations against use of the term "bio-identical" in marketing, as implying benefit for which there is no medical or scientific basis.

In the Study of Women's Health across the Nation [11], effects of hormone use were examined in 2,606 multiethnic women aged $42-52$ years followed for 5 years. The hormones examined were conjugated equine estrogen with or without a progestin. Hormone users had an increase in measure of oxidative stress, an increase in inflammatory response including an increase in C-reactive protein, and an increase in thrombotic activity. Challenge has been offered that these unfavorable hormone effects may have been mediated or influenced by the preexisting adverse coronary risk profiles of the women. These findings challenge the "timing hypothesis" which advocates for benefit of hormone therapy initiated early after menopause.

The timing hypothesis, i.e., that a favorable impact of menopausal hormone therapy on extending the advantageous premenopausal estrogen environment after menopause without a substantial discontinuity of time remains incompletely explored. A post hoc analysis in the WHI found a nonsignificant trend to benefit in women who initiated hormone therapy closer to menopause, and only the women in RUTH aged $<60$ years showed a significantly $(p=.003)$ lower incidence of coronary events in those assigned to raloxifene compared with placebo.

Nonetheless, the ACC/AHA clinical practice guidelines for coronary heart disease identify that hormone therapy and selective estrogen receptor modulators should not be used for the primary or secondary prevention of cardiovascular disease (level of evidence A) [12]. FDA labeling changes define that menopausal hormone therapy is not approved for heart disease prevention and may increase the risk for heart disease, heart attack, stroke, and breast cancer.

Has the last chapter been written on menopausal hormone therapy for cardioprotection - most certainly not. For example, can gene polymorphisms be used to identify hormone responders? Other unanswered questions include the effects of other formulations and doses of estrogen and progestin, the effect of non-oral routes of administration, and the effect of such therapy initiated earlier in menopause. This is being investigated in the KEEPS clinical trial, among others [13]. But, to provide an evidence base to inform health care decisions, such research clinical studies must have clinical outcome endpoints, not surrogate markers, and incorporate global risk scores to address the 
multisystem effects of menopausal hormone therapy. In the interim, clinicians are urged to aggressively identify, treat, and control established cardiovascular risk factors with an emphasis on therapeutic lifestyle changes.

\section{References}

1. Stampfer, J. M., \& Colditz, G. A. (1991). Estrogen replacement therapy and coronary heart disease: a quantitative assessment of the epidemiologic evidence. Preventive Medicine, 20(1), 47-63.

2. The Writing Group for the PEPI Trial. (1995). Effects of estrogen or estrogen/progestin regimens on heart disease risk factors in postmenopausal women: The Postmenopausal Estrogen/Progestin Interventions (PEPI) Trial. Journal of the American Medical Association, 273(3), 199-208.

3. Humphrey, L. L., Chan, B. K. S., \& Sox, H. C. (2002). Postmenopausal hormone replacement therapy and the primary prevention of cardiovascular disease. Annals of Internal Medicine, 137(4), 273-284.

4. Hulley, S., Grady, D., Bush, T., Furberg, C., Herrington, D., Riggs, B., et al. (1998). Randomized trial of estrogen plus progestin for secondary prevention of coronary heart disease in postmenopausal women. Journal of the American Medical Association, 280(7), 605-613.

5. Grady, D., Herrington, D., Bittner, V., Blumenthal, R., Davidson, M., Hlatky, M., et al. (2002). Cardiovascular disease outcomes during 6.8 years of hormone therapy. Heart and Estrogen/ Progestin Replacement Study Follow-up (HERS II). Journal of the American Medical Association, 288(1), 49-57.

6. Writing Group for the Women's Health Initiative Investigators. (2002). Risk and benefits of estrogen plus progestin in healthy postmenopausal women. Principal results from the Women's
Health Initiative Randomized Controlled Trial. Journal of the American Medical Association, 288(3), 321-333.

7. The Women's Health Initiative Steering Committee. (2004). Effects of conjugated equine estrogen in postmenopausal women with hysterectomy. The Women's Health Initiative Randomized Controlled Trial. Journal of the American Medical Association, 291(14), 1701-1712.

8. Shumaker, S. A., Legault, C., Rapp, S. R., Thal, L., Wallace, R. B., Ockene, J. K., et al. (2003). Estrogen plus progestin and the incidence of dementia and mild cognitive impairment in postmenopausal women. The Women's Health Initiative Memory Study: A randomized controlled trial. Journal of the American Medical Association, 289(20), 2651-2662.

9. Heiss, G., Wallace, R., Anderson, G. L., Aragaki, A., Beresford, S. A. A., Brzyski, R., et al. (2008). Health risks and benefits 3 years after stopping randomized treatment with estrogen and progestin. Journal of the American Medical Association, 299(9), 1036-1045.

10. Collins, P., Mosca, L., Geiger, M. J., Grady, D., Kornitzer, M., Amewou-Atisso, M. G., et al. (2009). Effects of the selective estrogen receptor modulator raloxifene on coronary outcomes in the Raloxifene Use for the Heart Trial. Results of subgroup analyses by age and other factors. Circulation, 119(7), 922-930.

11. Sowers, M. F., Randolph, J., Jr., Jannausch, M., Lasley, B., Jackson, E., \& McConnell, D. (2008). Levels of sex steroid and cardiovascular disease measures in premenopausal and hormonetreated women at midlife. Implications for the "Timing Hypothesis". Archives of Internal Medicine, 168(19), 2146-2153.

12. Mosca, L., Banks, C. L., Benjamin, E. J., Berra, K., Bushnell, C., Dolor, R. J., et al. (2007). Evidence-based guidelines for cardiovascular disease prevention in women: 2007 update. Journal of the American College of Cardiology, 49(11), 1230-1250.

13. Harman, S. M., Brinton, E. A., Cedars, M., Lobo, R., Manson, J. F., Merriam, G. R., et al. (2005). KEEPS: The Kronos Early Estrogen Prevention Trial. Climacteric, 8(1), 3-12. 